\title{
Diluting quark flavor hierarchies using dihedral symmetry
}

\author{
Ayushi Srivastava ${ }^{1, \mathrm{a}}$, Miguel Levy ${ }^{2, \mathrm{~b}} \mathbb{\mathbb { C }}$, Dipankar Das ${ }^{1, \mathrm{c}}$ \\ ${ }^{1}$ Department of Physics, Indian Institute of Technology (Indore), Khandwa Road, Simrol 453552, Indore, India \\ ${ }^{2}$ Departamento de Física, Centro de Física Teórica de Partículas-CFTP, Instituto Superior Técnico, Universidade de Lisboa, Av Rovisco Pais, 1 , \\ 1049-001 Lisboa, Portugal
}

Received: 20 October 2021 / Accepted: 11 February 2022 / Published online: 8 March 2022

(C) The Author(s) 2022

\begin{abstract}
We present a $D_{4}$ flavored extension of the SM which provides an intuitive reasoning for the masses and mixing patterns in the quark sector. In our model, the Cabibbo mixing angle becomes related to the ratio of two vacuum expectation values. In fact, the orders of magnitude of the CKM matrix elements are readily obtained from the hierarchical nature of the vacuum expectation values. Moreover, we also show that the smallness of the off-Cabibbo elements in the CKM matrix is strongly connected to the heaviness of the third generation of quarks.

The Standard Model (SM) successfully explains the mechanism responsible for the fermion masses but does not justify them. The arbitrariness of the Yukawa couplings makes the SM adaptable to any spectrum of fermion masses and mixings brought in by the experimental measurements. As it happens, the observed quark masses span five orders of magnitudes, with the third generation of quarks being much heavier than the first two. Furthermore, the quark mixings obey the following hierarchical pattern [1]:
\end{abstract}

$V \approx\left(\begin{array}{ccc}1-\lambda^{2} / 2 & -\lambda & \mathcal{O}\left(\lambda^{3}\right) \\ \lambda & 1-\lambda^{2} / 2 & \mathcal{O}\left(\lambda^{2}\right) \\ \mathcal{O}\left(\lambda^{3}\right) & \mathcal{O}\left(\lambda^{2}\right) & 1\end{array}\right)$

where $\lambda \approx 0.22$ is the Cabibbo mixing parameter and the matrix, $V$, is known as the Cabibbo-Kobayashi-Maskawa (CKM) matrix [2-4]. Within the ambit of SM, such hierarchies can only originate from some conspiracies within the Yukawa couplings themselves [5]. This is part of the problem that is usually dubbed in the literature as the 'flavor puzzle' [6-8]. This aspect of the SM, for decades, has fueled speculations that there might exist a deeper theoretical framework which can offer a more natural insight into the flavor structure. This article presents an extension beyond the SM (BSM) with a $D_{4}$ symmetry, which can make the quark flavor structure appear more instinctive (for other works on flavor models using $D_{4}$ symmetry, see [9-17]). An essential ingredient of our model is that the primary sources of masses for the third generation of quarks have been disentangled from those for the first two generations of quarks. The hierarchies in the quark masses and mixings are then chiefly attributed to the hierarchies in the vacuum expectation values (VEVs) of the different scalar fields. This allows us to relax the Yukawa hierarchies in the quark sector considerably along with some new and interesting implications for the CKM matrix. We will describe our model in detail in the upcoming sections.

We start by laying out some of the basics of $D_{4}$ symmetry [18]. The discrete group $D_{4}$ has five irreducible representations which we label as $\mathbf{1}_{++}, \mathbf{1}_{--}, \mathbf{1}_{-+}, \mathbf{1}_{+-}$, and $\mathbf{2}$. For the two-dimensional representation of $D_{4}$, we opt to work in a basis in which the generators of $D_{4}$ are given by

$a=\left[\begin{array}{cc}0 & -1 \\ 1 & 0\end{array}\right], \quad b=\left[\begin{array}{cc}1 & 0 \\ 0 & -1\end{array}\right]$,

where $a$ is of order 4 and $b$ is of order 2. In this basis, the relevant tensor products in the explicit component form are given by [19]

$$
\begin{aligned}
{\left[\begin{array}{l}
x_{1} \\
x_{2}
\end{array}\right]_{\mathbf{2}} \otimes\left[\begin{array}{l}
y_{1} \\
y_{2}
\end{array}\right]_{\mathbf{2}}=} & {\left[x_{1} y_{1}+x_{2} y_{2}\right]_{\mathbf{1}_{++}} \oplus\left[x_{1} y_{2}-x_{2} y_{1}\right]_{\mathbf{1}_{--}} } \\
& \oplus\left[x_{1} y_{2}+x_{2} y_{1}\right]_{\mathbf{1}_{-+}} \\
& \oplus\left[x_{1} y_{1}-x_{2} y_{2}\right]_{\mathbf{1}_{+-}} \\
\mathbf{1}_{r, s} \otimes \mathbf{1}_{r^{\prime}, s^{\prime}}= & \mathbf{1}_{r \cdot r^{\prime}, s \cdot s^{\prime}} .
\end{aligned}
$$

Now we will specify the $D_{4}$ transformations of the different fields in our model. The $i$-th generation of left-handed quark doublet is denoted by $Q_{i L} \equiv\left(p_{i L}, n_{i L}\right)^{T}$. The right-handed

\footnotetext{
a e-mail: srivastavaayushi860@gmail.com

b e-mail: miguelplevy@ist.utl.pt (corresponding author)

ce-mail: d.das@iiti.ac.in
} 
charged quark singlets are denoted by $p_{i R}$ and $n_{i R}$ in the up and down sectors, respectively. We have four scalar doublets in our model, which we symbolize as $\phi_{1}, \phi_{2}, \phi_{u}$ and $\phi_{d}$. These fields are assumed to transform under the $D_{4}$ symmetry as follows:

$$
\begin{aligned}
& \mathbf{2}:\left[\begin{array}{c}
Q_{1 L} \\
Q_{2 L}
\end{array}\right],\left[\begin{array}{c}
\phi_{1} \\
\phi_{2}
\end{array}\right], \\
& \mathbf{1}_{++}: n_{1 R}, \mathbf{1}_{--}: n_{2 R}, n_{3 R}, \phi_{u}, \mathbf{1}_{-+}: p_{2 R}, p_{3 R}, \phi_{d}, \\
& \mathbf{1}_{+-}: Q_{3 L}, p_{1 R} .
\end{aligned}
$$

As we will see shortly, because of the above transformations, $\phi_{u}$ and $\phi_{d}$ will couple exclusively to the up and down type quarks respectively, which justifies their labeling. The gauge and $D_{4}$ invariant Yukawa Lagrangians in the up and down quark sectors are then given by

$$
\begin{gathered}
M_{u} M_{u}^{\dagger}=\left(\begin{array}{ccc}
A_{u}^{2} v_{1}^{2}+\left(B_{u}^{2}+C_{u}^{2}\right) v_{2}^{2} & \left(-A_{u}^{2}+B_{u}^{2}+C_{u}^{2}\right) v_{1} v_{2} & \left(C_{u} Y_{u}+B_{u} X_{u}\right) v_{2} v_{u} \\
\left(-A_{u}^{2}+B_{u}^{2}+C_{u}^{2}\right) v_{1} v_{2} & \left(B_{u}^{2}+C_{u}^{2}\right) v_{1}^{2}+A_{u}^{2} v_{2}^{2} & \left(C_{u} Y_{u}+B_{u} X_{u}\right) v_{1} v_{u} \\
\left(C_{u} Y_{u}+B_{u} X_{u}\right) v_{2} v_{u} & \left(C_{u} Y_{u}+B_{u} X_{u}\right) v_{1} v_{u} & \left(Y_{u}^{2}+X_{u}^{2}\right) v_{u}^{2}
\end{array}\right), \\
M_{d} M_{d}^{\dagger}=\left(\begin{array}{ccc}
A_{d}^{2} v_{1}^{2}+\left(B_{d}^{2}+C_{d}^{2}\right) v_{2}^{2} & \left(A_{d}^{2}-B_{d}^{2}-C_{d}^{2}\right) v_{1} v_{2} & \left(C_{d} Y_{d}+B_{d} X_{d}\right) v_{2} v_{d} \\
\left(A_{d}^{2}-B_{d}^{2}-C_{d}^{2}\right) v_{1} v_{2} & \left(B_{d}^{2}+C_{d}^{2}\right) v_{1}^{2}+A_{d}^{2} v_{2}^{2} & -\left(C_{d} Y_{d}+B_{d} X_{d}\right) v_{1} v_{d} \\
\left(C_{d} Y_{d}+B_{d} X_{d}\right) v_{2} v_{d} & -\left(C_{d} Y_{d}+B_{d} X_{d}\right) v_{1} v_{d} & \left(Y_{d}^{2}+X_{d}^{2}\right) v_{d}^{2}
\end{array}\right) .
\end{gathered}
$$

$$
\begin{aligned}
-\mathscr{L}_{u}= & A_{u}\left(\bar{Q}_{1 L} \widetilde{\phi}_{1}-\bar{Q}_{2 L} \widetilde{\phi}_{2}\right) p_{1 R}+B_{u}\left(\bar{Q}_{1 L} \widetilde{\phi}_{2}\right. \\
& \left.+\bar{Q}_{2 L} \widetilde{\phi}_{1}\right) p_{2 R}+C_{u}\left(\bar{Q}_{1 L} \widetilde{\phi}_{2}+\bar{Q}_{2 L} \widetilde{\phi}_{1}\right) p_{3 R} \\
& +X_{u} \bar{Q}_{3 L} \phi_{u} p_{2 R} \\
& +Y_{u} \bar{Q}_{3 L} \phi_{u} p_{3 R}, \\
-\mathscr{L}_{d}= & A_{d}\left(\bar{Q}_{1 L} \phi_{1}+\bar{Q}_{2 L} \phi_{2}\right) n_{1 R} \\
& +B_{d}\left(\bar{Q}_{1 L} \phi_{2}-\bar{Q}_{2 L} \phi_{1}\right) n_{2 R} \\
& +C_{d}\left(\bar{Q}_{1 L} \phi_{2}-\bar{Q}_{2 L} \phi_{1}\right) n_{3 R} \\
& +X_{d} \bar{Q}_{3 L} \phi_{d} n_{2 R}+Y_{d} \bar{Q}_{3 L} \phi_{d} n_{3 R},
\end{aligned}
$$

where $\widetilde{\phi}_{k}=i \sigma_{2} \phi_{k}^{\star}$ with $\sigma_{2}$ being the second Pauli matrix. For an intuitive understanding of the upcoming results, we will assume the Yukawa parameters to be real. As such, we will not deliberate so much on the complex phase of the CKM matrix. We will treat the phase as an independent parameter which, as we have checked, can be easily accommodated by allowing the Yukawa couplings to be complex. The mass matrices in the up and down sector that transpire from Eq. (5) are

$$
\begin{aligned}
M_{u} & =\left(\begin{array}{ccc}
A_{u} v_{1} & B_{u} v_{2} & C_{u} v_{2} \\
-A_{u} v_{2} & B_{u} v_{1} & C_{u} v_{1} \\
0 & X_{u} v_{u} & Y_{u} v_{u}
\end{array}\right), \\
M_{d} & =\left(\begin{array}{ccc}
A_{d} v_{1} & B_{d} v_{2} & C_{d} v_{2} \\
A_{d} v_{2} & -B_{d} v_{1} & -C_{d} v_{1} \\
0 & X_{d} v_{d} & Y_{d} v_{d}
\end{array}\right),
\end{aligned}
$$

where $v_{1}, v_{2}, v_{u}$ and $v_{d}$ represents the VEVs of $\phi_{1}, \phi_{2}, \phi_{u}$ and $\phi_{d}$ respectively with the total electroweak $\mathrm{VEV}, v$, being defined through the relation

$v^{2}=v_{1}^{2}+v_{2}^{2}+v_{u}^{2}+v_{d}^{2} \approx(174 \mathrm{GeV})^{2}$.

The diagonal mass matrices can then be obtained via the following biunitary transformations:

$D_{u}=U_{u} M_{u} V_{u}^{\dagger}=\operatorname{diag}\left(m_{u}, m_{c}, m_{t}\right)$,

$D_{d}=U_{d} M_{d} V_{d}^{\dagger}=\operatorname{diag}\left(m_{d}, m_{s}, m_{b}\right)$.

Following this convention for the biunitary transformations, the CKM matrix will be given by

$V_{\mathrm{CKM}}=U_{u} U_{d}^{\dagger}$.

The matrices $U_{u}$ and $U_{d}$ are obtained by diagonalizing $M_{u} M_{u}^{\dagger}$ and $M_{d} M_{d}^{\dagger}$ respectively, which can be calculated from Eq. (6) as follows:

As a matter of fact, both $M_{u} M_{u}^{\dagger}$ and $M_{d} M_{d}^{\dagger}$ can be fully diagonalized analytically by sequentially operating the following matrices:

$$
\begin{aligned}
O_{\beta} & =\left(\begin{array}{ccc}
\cos \beta & -\sin \beta & 0 \\
\sin \beta & \cos \beta & 0 \\
0 & 0 & 1
\end{array}\right), \\
O_{\theta}^{u, d} & =\left(\begin{array}{ccc}
1 & 0 & 0 \\
0 & \cos \theta_{u, d} & -\sin \theta_{u, d} \\
0 & \sin \theta_{u, d} & \cos \theta_{u, d}
\end{array}\right),
\end{aligned}
$$

where $\tan \beta=v_{2} / v_{1}$ and $\theta_{u, d}$ will be defined shortly. As a first step, we notice that $M_{u} M_{u}^{\dagger}$ and $M_{d} M_{d}^{\dagger}$ can be block diagonalized using $O_{\beta}$ as

$$
\begin{aligned}
& \left(M_{u}^{2}\right)_{\text {Block }} \equiv O_{\beta} M_{u} M_{u}^{\dagger} O_{\beta}^{\dagger} \\
& =\left(\begin{array}{ccc}
A_{u}^{2} v_{12}^{2} & 0 & 0 \\
0 & \left(B_{u}^{2}+C_{u}^{2}\right) v_{12}^{2} & \left(C_{u} Y_{u}+B_{u} X_{u}\right) v_{12} v_{u} \\
0 & \left(C_{u} Y_{u}+B_{u} X_{u}\right) v_{12} v_{u} & \left(Y_{u}^{2}+X_{u}^{2}\right) v_{u}^{2}
\end{array}\right), \\
& \left(M_{d}^{2}\right)_{\text {Block }} \equiv O_{\beta}^{\dagger} M_{d} M_{d}^{\dagger} O_{\beta} \\
& =\left(\begin{array}{ccc}
A_{d}^{2} v_{12}^{2} & 0 & 0 \\
0 & \left(B_{d}^{2}+C_{d}^{2}\right) v_{12}^{2} & -\left(C_{d} Y_{d}+B_{d} X_{d}\right) v_{12} v_{d} \\
0 & -\left(C_{d} Y_{d}+B_{d} X_{d}\right) v_{12} v_{d} & \left(Y_{d}^{2}+X_{d}^{2}\right) v_{d}^{2}
\end{array}\right),
\end{aligned}
$$

where, as we will see shortly, $v_{12}^{2}=v_{1}^{2}+v_{2}^{2}$ is the total $\mathrm{VEV}$ that is primarily responsible for the light quark masses. Quite clearly, the remaining $2 \times 2$ block in the up and down 
sectors can be diagonalized using $O_{\theta}^{u}$ and $O_{\theta}^{d}$, respectively. This second stage of diagonalization allows us to express $\theta_{u}$ and $\theta_{d}$ in terms of the Yukawa couplings and the VEVs as follows:

$$
\begin{aligned}
\tan 2 \theta_{u} & =\frac{2\left(C_{u} Y_{u}+B_{u} X_{u}\right) v_{12} v_{u}}{\left(Y_{u}^{2}+X_{u}^{2}\right) v_{u}^{2}-\left(B_{u}^{2}+C_{u}^{2}\right) v_{12}^{2}}, \\
\tan 2 \theta_{d} & =-\frac{2\left(C_{d} Y_{d}+B_{d} X_{d}\right) v_{12} v_{d}}{\left(Y_{d}^{2}+X_{d}^{2}\right) v_{d}^{2}-\left(B_{d}^{2}+C_{d}^{2}\right) v_{12}^{2}} .
\end{aligned}
$$

Thus, the full diagonalization in the up and down sectors can be expressed as

$$
\begin{aligned}
& D_{u}^{2}=O_{\theta}^{u} O_{\beta}\left(M_{u} M_{u}^{\dagger}\right) O_{\beta}^{\dagger} O_{\theta}^{u \dagger} \equiv \operatorname{diag}\left(m_{u}^{2}, m_{c}^{2}, m_{t}^{2}\right), \\
& D_{d}^{2}=O_{\theta}^{d} O_{\beta}^{\dagger}\left(M_{d} M_{d}^{\dagger}\right) O_{\beta} O_{\theta}^{d^{\dagger}} \equiv \operatorname{diag}\left(m_{d}^{2}, m_{s}^{2}, m_{b}^{2}\right) .
\end{aligned}
$$

Following our convention in Eq. (8), the matrices $U_{u}$ and $U_{d}$ can be extracted as follows:

$U_{u}=O_{\theta}^{u} O_{\beta}, \quad U_{d}=O_{\theta}^{d} O_{\beta}^{\dagger}$.

Thus from Eq. (9), the CKM matrix is obtained as then Eq. (16) resembles exactly to Eq. (1). All these intuitive results will be validated later by providing explicit numerical benchmarks.

Given the structure of the CKM matrix predicted by the model as a function of $\beta$ and $\theta_{u, d}$, shown in Eq. (16), it is possible to extract the quark mixing angles by comparing the CKM matrix with the standard parametrization [20]. This, in turn, allows us to find the following best-fit values of $\beta$ and $\theta_{u, d}$ such that the quark mixing angles are compatible with the observed values [21]:

$\sin 2 \beta \approx 0.2265, \quad \theta_{u} \approx \pm 0.025, \quad \theta_{d} \approx \mp 0.016$.

As expected, the above values for $\sin 2 \beta$ and $\theta_{u, d}$ conform well to our intuitive expectations of Eqs. (19) and (20). Fixing $\sin 2 \beta$ at its best-fit value, in Fig. 1 we display the region in $\sin \theta_{u}$ - $\sin \theta_{d}$ plane allowed by the experimental uncertainties.

$V_{\mathrm{CKM}}=\left(\begin{array}{ccc}\cos 2 \beta & -\cos \theta_{d} \sin 2 \beta & -\sin 2 \beta \sin \theta_{d} \\ \cos \theta_{u} \sin 2 \beta & \cos 2 \beta \cos \theta_{d} \cos \theta_{u}+\sin \theta_{d} \sin \theta_{u} & \cos 2 \beta \cos \theta_{u} \sin \theta_{d}-\cos \theta_{d} \sin \theta_{u} \\ \sin 2 \beta \sin \theta_{u} & -\cos \theta_{u} \sin \theta_{d}+\cos 2 \beta \cos \theta_{d} \sin \theta_{u} & \cos \theta_{d} \cos \theta_{u}+\cos 2 \beta \sin \theta_{d} \sin \theta_{u}\end{array}\right)$.

To make the connection between Eqs. (16) and (1) apparent, we assume that $v_{12}$ is responsible for the masses of the first two generations of quarks whereas $v_{u}$ and $v_{d}$ primarily contribute to the third generation masses in the up and down sector, respectively. Therefore, it is quite natural to expect $v_{12} \ll v_{u, d}$. From Eq. (12) we identify the first generation quark masses as

$m_{u}^{2}=A_{u}^{2} v_{12}^{2}, \quad m_{d}^{2}=A_{d}^{2} v_{12}^{2}$.

Furthermore, using the VEV hierarchy $v_{u, d} \gg v_{12}$ we can approximate Eq. (13) as

$\theta_{u} \approx \frac{\left(C_{u} Y_{u}+B_{u} X_{u}\right)}{\left(Y_{u}^{2}+X_{u}^{2}\right)} \frac{v_{12}}{v_{u}} \approx \mathcal{O}\left(\frac{v_{12}}{v_{u}}\right)$,

$\theta_{d} \approx-\frac{\left(C_{d} Y_{d}+B_{d} X_{d}\right)}{\left(Y_{d}^{2}+X_{d}^{2}\right)} \frac{v_{12}}{v_{d}} \approx \mathcal{O}\left(\frac{v_{12}}{v_{d}}\right)$,

where we are implicitly assuming that the involved Yukawa couplings have similar orders of magnitude. It is also quite reasonable to take $v_{12} \sim \mathcal{O}(1 \mathrm{GeV})$ and $v_{u, d} \sim$ $\mathcal{O}(100 \mathrm{GeV})$ so that the ratio $v_{12} / v_{u, d}$ comes out to be $\mathcal{O}\left(\lambda^{2}\right)$. Therefore, from Eq. (18) we conclude

$\sin \theta_{u, d} \approx \mathcal{O}\left(\lambda^{2}\right), \quad \cos \theta_{u, d} \approx \mathcal{O}(1)$.

Moreover, if we identify $\sin 2 \beta$ as the Cabibbo mixing, namely,

$\sin 2 \beta=\lambda$,
For the sake of completeness, we also calculate the mass eigenvalues for the second and third generation of quarks by diagonalizing the $2 \times 2$ submatrices in Eq. (12). In the up quark sector, we can compare the traces to write

$m_{c}^{2}+m_{t}^{2}=\left(B_{u}^{2}+C_{u}^{2}\right) v_{12}^{2}+\left(Y_{u}^{2}+X_{u}^{2}\right) v_{u}^{2}$.

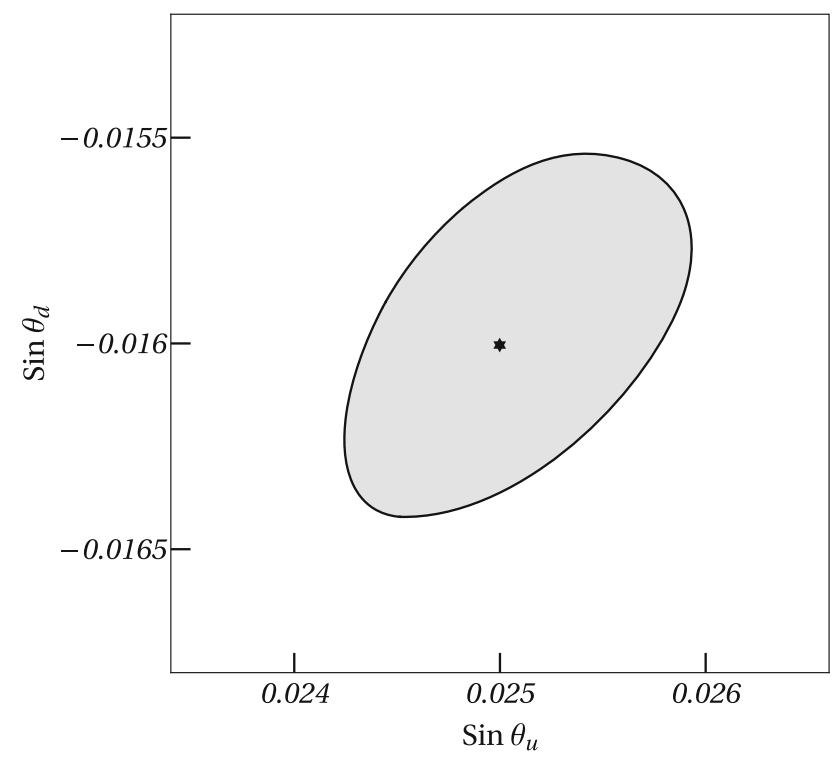

Fig. 1 A representative allowed region in the $\sin \theta_{u}$ - $\sin \theta_{d}$ plane from the uncertainties in $\theta_{23}$ and $\theta_{13}$. For this plot, we have fixed $\sin 2 \beta$ at its best-fit value given in Eq. (21). The best-fit point in the $\sin \theta_{u}$ - $\sin \theta_{d}$ plane is marked with a star $(\star)$ 
Keeping in mind the hierarchies, $v_{u} \gg v_{12}$ and $m_{t} \gg m_{c}$, the above relation can be approximated to express the top quark mass as

$m_{t}^{2} \approx\left(Y_{u}^{2}+X_{u}^{2}\right) v_{u}^{2}$.

Again, from the determinant of the $2 \times 2$ block in Eq. (12a), we may write

$m_{c}^{2} m_{t}^{2}=\left(B_{u} Y_{u}-C_{u} X_{u}\right)^{2} v_{12}^{2} v_{u}^{2}$.

Using the expression for $m_{t}$ from Eq. (23), we can extract the charm quark mass as

$m_{c}^{2} \approx \frac{\left(B_{u} Y_{u}-C_{u} X_{u}\right)^{2}}{\left(Y_{u}^{2}+X_{u}^{2}\right)} v_{12}^{2}$.

Following the same steps in the down sector, we can obtain

$m_{s}^{2} \approx \frac{\left(B_{d} Y_{d}-C_{d} X_{d}\right)^{2}}{\left(Y_{d}^{2}+X_{d}^{2}\right)} v_{12}^{2}$,

$m_{b}^{2} \approx\left(Y_{d}^{2}+X_{d}^{2}\right) v_{d}^{2}$.

At this point, we wish to emphasize that, assuming the Yukawas couplings to be similar for a particular sector, an obvious outcome of our model is

$\frac{m_{c}}{m_{t}} \approx \frac{v_{12}}{v_{u}} \sim \mathcal{O}\left(\lambda^{2}\right), \quad \frac{m_{s}}{m_{b}} \approx \frac{v_{12}}{v_{d}} \sim \mathcal{O}\left(\lambda^{2}\right)$,

which agrees with the observations.

From Eqs. (23) and (25), we see that the third and second generation masses are controlled by $v_{u}$ and $v_{12}$, respectively. We can wonder how perturbativity may affect the model at hand, since $m_{t} \approx \mathcal{O}\left(v_{u}\right)$ and $m_{c} \approx \mathcal{O}\left(v_{12}\right)$ already [22]. Figure 2 illustrates how arbitrarily low values of $v_{12}$ may jeopardize the perturbativity of the theory. By choosing $v_{u}=$ $150 \mathrm{GeV}$, and two example values for $X_{u}=0.7$ and 0.9 , we can see from Fig. 2 that to have $B_{u}$ and $C_{u}$ in the perturbative regime, we should have $v_{12} \geq \mathcal{O}(1 \mathrm{GeV})$.

Finally, to provide explicit justification to these intuitive expectations, we consider the following benchmark

$$
\begin{array}{lll}
v_{12}=2 \mathrm{GeV}, & v_{u}=150 \mathrm{GeV}, & v_{d} \approx 88 \mathrm{GeV}, \\
A_{u} \approx 1.08 \times 10^{-3}, B_{u} \approx 1.69, & C_{u} \approx 1.50, \\
X_{u} \approx 1.04, & Y_{u} \approx 0.49, A_{d} \approx 2.34 \times 10^{-3}, & B_{d} \approx 3.65 \times 10^{-2}, \\
C_{d} \approx 4.41 \times 10^{-2}, & & \\
X_{d} \approx 4.73 \times 10^{-2}, Y_{d} \approx-3.20 \times 10^{-3}, & &
\end{array}
$$

which results in the following values of the quark masses and mixing angles

$$
\begin{aligned}
& m_{u}=2.2 \mathrm{MeV}, \quad m_{c}=1.27 \mathrm{GeV}, \quad m_{t}=173 \mathrm{GeV}, \\
& m_{d}=4.7 \mathrm{MeV}, \quad m_{s}=0.093 \mathrm{GeV}, \quad m_{b}=4.18 \mathrm{GeV} \\
& \sin \theta_{12}=0.2265, \quad \sin \theta_{13}=0.0036, \quad \sin \theta_{23}=0.041,
\end{aligned}
$$

which are in agreement with the corresponding observations [21].

In passing, let us highlight the most notable outcomes of our model:

- The hierarchy of the Yukawa couplings is diluted by two orders of magnitude, at least. Recall that, in the SM, $m_{t}=174 \mathrm{GeV}$ and $m_{u, d} \sim \mathcal{O}\left(10^{-3} \mathrm{GeV}\right)$ imply that the quark Yukawa couplings span five orders of magnitudes. We dampen this problem by assuming that the first two generations of quarks receive their masses from $v_{12}$ which is of $\mathcal{O}(1 \mathrm{GeV})$. This means, the first generation Yukawas are, at worst, of $\mathcal{O}\left(10^{-3}\right)$ whereas the second generation Yukawas can be of $\mathcal{O}(1)$. This feature is quite evident from the benchmark values given in Eq. (29).

- We have introduced $\phi_{u, d}$ dedicated for masses of the third generation of quarks. Quite naturally, we expect, $v_{u, d} \sim$ $\mathcal{O}(100 \mathrm{GeV})$ so that the top-Yukawa is of $\mathcal{O}(1)$. Thus, we should have the ratio $v_{12} / v_{u, d} \sim \mathcal{O}\left(\lambda^{2}\right)$. It is very interesting to note that, this automatically conforms to $m_{2} / m_{3} \approx v_{12} / v_{u, d} \sim \mathcal{O}\left(\lambda^{2}\right)$ where $m_{k}$ is the mass for the $k$-th generation of quark. Quite clearly, this is a natural upshot of our model.

- We have connected the quark mixings with the dynamics of the scalar sector. We have shown that the Cabibbo part of the quark-mixing stems purely from the ratio $v_{2} / v_{1}$ (see Eq. (20)). The smallness of the off-Cabibbo elements of the CKM matrix is further connected to the VEV hierarchy $v_{12} \ll v_{u, d}$. In other way, we are suggesting that the fact that the third generation of quarks are much heavier than the first two generations, is intimately connected to the smallness of the off-Cabibbo elements.

Finally, our current model is not just all about aesthetics, it will have other observable consequences too. The fact that the Yukawa Lagrangian of our model contains fewer parameters than that of the SM comes at the cost of making the scalar potential substantially more involved containing four scalar doublets. This means that the Higgs boson observed at the LHC is not the only fundamental scalar in nature, it is just the first one in series of many others to follow. The physical Higgs bosons will emerge from mixings among the four scalar doublets. Expanding the scalar doublets as

$\phi_{k}=\left(\begin{array}{c}\varphi_{k}^{+} \\ v_{k}+\left(h_{k}+i z_{k}\right) / \sqrt{2}\end{array}\right), \quad k=1,2, u, d$,

after the spontaneous symmetry breaking, the SM-like Higgs boson, $h$, can be extracted as follows [23]:

$h=\frac{1}{v}\left(v_{1} h_{1}+v_{2} h_{2}+v_{u} h_{u}+v_{d} h_{d}\right)$.

This particular linear combination of the component fields will mimic the SM Higgs in its tree-level couplings and will 


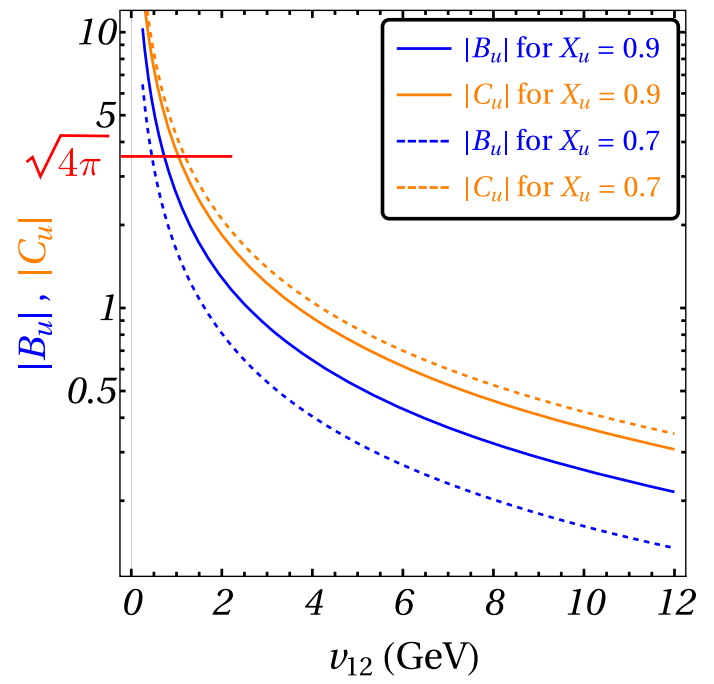

Fig. 2 Left: Values of $\left|B_{u}\right|$ (blue) and $\left|C_{u}\right|$ (orange) compatible with the experimental values for the quark masses and mixing angles, for the benchmark points of $X_{u}=0.9$ (solid line) and $X_{u}=0.7$ (dashed), as

not induce flavor changing neutral currents (FCNCs) at the tree-level. However, the other physical neutral scalars, in general, will possess tree-level FCNCs which means they have to be quite heavy to evade the experimental constraints. To have some intuitions on the FCNC couplings, we analyze the matrices, $N_{d}^{1,2, d}$, which control them in the down sector. Below, we show approximate expressions for these matrices:

$$
\begin{aligned}
& N_{d}^{1} \approx \frac{1}{\sqrt{2} v_{12}}\left(\begin{array}{ccc}
m_{d} \cos \beta & -m_{s} \sin \beta & m_{b} \theta_{d} \sin \beta \\
-m_{d} \sin \beta & -m_{s} \cos \beta & m_{b} \theta_{d} \cos \beta \\
-m_{d} \theta_{d} \sin \beta & -m_{s} \theta_{d} \cos \beta & m_{b} \theta_{d}^{2} \cos \beta
\end{array}\right), \\
& N_{d}^{2} \approx \frac{1}{\sqrt{2} v_{12}}\left(\begin{array}{ccc}
m_{d} \sin \beta & m_{s} \cos \beta & -m_{b} \theta_{d} \cos \beta \\
m_{d} \cos \beta & -m_{s} \sin \beta & m_{b} \theta_{d} \sin \beta \\
m_{b} \theta_{d} \cos \beta & -m_{s} \theta_{d} \sin \beta & m_{b} \theta_{d}^{2} \sin \beta
\end{array}\right), \\
& N_{d}^{d} \approx \frac{1}{\sqrt{2} v_{d}}\left(\begin{array}{ccc}
0 & 0 & 0 \\
0 & 0 & -m_{b} \theta_{d} \\
0 & 0 & m_{b}
\end{array}\right)
\end{aligned}
$$

From the above expressions, we note that the magnitude of the largest off-diagonal element, for our chosen benchmarks of Eqs. (21) and (29), is 0.033, which is quite small. On top of this, the flavor constraints may be further relaxed if we remember the following points:

- The actual FCNC matrices that control the couplings of the physical neutral scalars are orthogonal linear combinations (dictated by the scalar potential) of $N_{d}^{1}, N_{d}^{2}$, $N_{d}^{d}$ and $N_{d}^{u}$ where $N_{d}^{u}=0$ simply because $h_{u}$ does not couple to the down-type quarks.

- A cancellation may be arranged between the scalar and pseudoscalar diagrams appearing in the FCNC process [24].

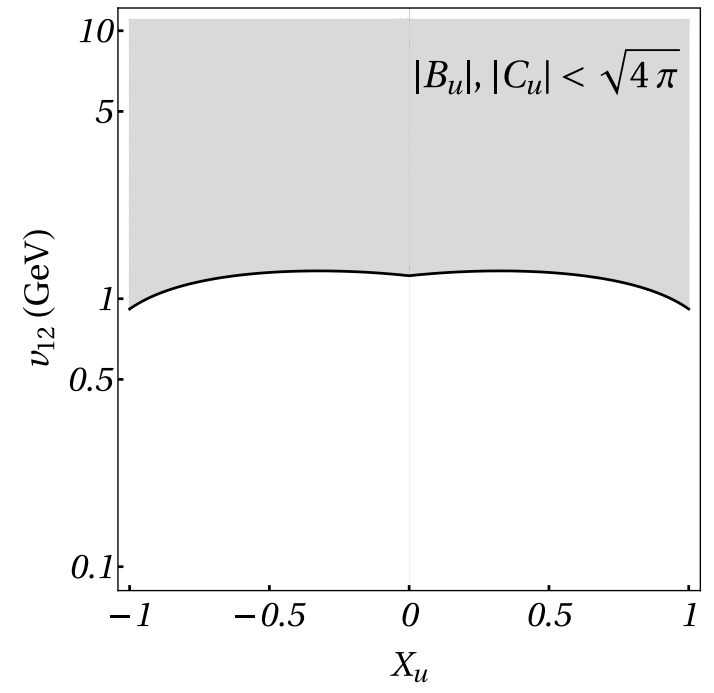

a function of $v_{12}$. The perturbative bound is marked in red. Right: $\left|B_{u}\right|$ and $\left|C_{u}\right|$ are perturbative in the shaded region in the $X_{u}-v_{12}$ plane. We have assumed $v_{u}=150 \mathrm{GeV}$ and $\sin \theta_{u}=0.025$ for both cases

Furthermore, it should also be noted that low values of the VEVs, especially $v_{12} \sim \mathcal{O}(1 \mathrm{GeV})$, will not necessarily imply the existence of light nonstandard scalars if we include terms that softly break the $D_{4}$ symmetry in the scalar potential [25-27] (please refer to the appendix A for details). A detailed study of the scalar potential along with the analysis of the flavor constraints is reserved for a future work. Nevertheless, our current paper can be considered as a proof-ofconcept for a novel idea that it might be possible to ascribe the quark flavor hierarchies primarily to the hierarchies in the VEVs all of which add together to constitute the total electroweak $\mathrm{VEV}^{1}$. Thus, in other words, we have demonstrated that, to have an insight into the flavor puzzle, it might not be necessary to appeal to flavor symmetry breaking scales much higher than the EW scale. Moreover, the fact that such a scheme can be easily accommodated in a relatively simple and intuitive theoretical set-up, makes our model an interesting addition to the existing literature on flavor model building.

Acknowledgements We thank Palash B. Pal for useful insights about the model. We thank Ipsita Saha for help with Mathematica. DD thanks the Science and Engineering Research Board, India for financial support through grant no. SRG/2020/000006. The work of ML is funded by Fundação para a Ciência e Tecnologia-FCT through Grant No.PD/BD/150488/2019, in the framework of the Doctoral Programme IDPASC-PT, and in part by the projects CFTP-FCT Unit 777 (UIDB/00777/2020 and UIDP/00777/2020), and CERN/FISPAR/0008/2019.

1 This is in stark contrast with the Froggatt-Nielsen mechanism [28],
where flavon VEVs are usually much higher than the electroweak scale. 
Data Availability Statement This manuscript has no associated data or the data will not be deposited. [Authors' comment: Since this is a theoretical research, there is no data.]

Open Access This article is licensed under a Creative Commons Attribution 4.0 International License, which permits use, sharing, adaptation, distribution and reproduction in any medium or format, as long as you give appropriate credit to the original author(s) and the source, provide a link to the Creative Commons licence, and indicate if changes were made. The images or other third party material in this article are included in the article's Creative Commons licence, unless indicated otherwise in a credit line to the material. If material is not included in the article's Creative Commons licence and your intended use is not permitted by statutory regulation or exceeds the permitted use, you will need to obtain permission directly from the copyright holder. To view a copy of this licence, visit http://creativecomm ons.org/licenses/by/4.0/.

Funded by SCOAP ${ }^{3}$.

\section{A Discussion of the scalar potential}

It might be natural to wonder whether the VEV hierarchies and the scalar mass spectrum required for our model to work are admissible by the scalar sector of our model obeying the $D_{4}$ symmetry. Here, we will explicitly demonstrate that such features can indeed be achieved when the scalar potential contains terms that softly-break the symmetry. In view of this, let us write down the scalar potential as follows:

$V(\phi)=V_{\text {quadratic }}+V_{\text {quartic }}$,

where,

$$
\begin{aligned}
V_{\text {quartic }}= & \lambda_{1}\left(\phi_{1}^{\dagger} \phi_{1}+\phi_{2}^{\dagger} \phi_{2}\right)^{2}+\lambda_{2}\left(\phi_{1}^{\dagger} \phi_{2}-\phi_{2}^{\dagger} \phi_{1}\right)^{2} \\
& +\lambda_{3}\left(\phi_{1}^{\dagger} \phi_{2}+\phi_{2}^{\dagger} \phi_{1}\right)^{2}+\lambda_{4}\left(\phi_{1}^{\dagger} \phi_{1}-\phi_{2}^{\dagger} \phi_{2}\right)^{2} \\
& +\lambda_{5}\left(\phi_{u}^{\dagger} \phi_{u}\right)^{2}+\lambda_{6}\left(\phi_{d}^{\dagger} \phi_{d}\right)^{2} \\
& +\lambda_{7}\left(\phi_{1}^{\dagger} \phi_{1}+\phi_{2}^{\dagger} \phi_{2}\right)\left(\phi_{d}^{\dagger} \phi_{d}\right) \\
& +\lambda_{8}\left(\phi_{1}^{\dagger} \phi_{1}+\phi_{2}^{\dagger} \phi_{2}\right)\left(\phi_{u}^{\dagger} \phi_{u}\right) \\
& +\lambda_{9}\left(\phi_{u}^{\dagger} \phi_{u}\right)\left(\phi_{d}^{\dagger} \phi_{d}\right) \\
& +\lambda_{10}\left[\left(\phi_{u}^{\dagger} \phi_{1}\right)\left(\phi_{1}^{\dagger} \phi_{u}\right)+\left(\phi_{u}^{\dagger} \phi_{2}\right)\left(\phi_{2}^{\dagger} \phi_{u}\right)\right] \\
& +\lambda_{11}\left[\left(\phi_{d}^{\dagger} \phi_{1}\right)\left(\phi_{1}^{\dagger} \phi_{d}\right)+\left(\phi_{d}^{\dagger} \phi_{2}\right)\left(\phi_{2}^{\dagger} \phi_{d}\right)\right] \\
& +\left\{\lambda_{12}\left[\left(\phi_{u}^{\dagger} \phi_{1}\right)^{2}+\left(\phi_{u}^{\dagger} \phi_{2}\right)^{2}\right]\right. \\
& +\lambda_{13}\left[\left(\phi_{d}^{\dagger} \phi_{1}\right)^{2}+\left(\phi_{d}^{\dagger} \phi_{2}\right)^{2}\right] \\
& +\lambda_{14}\left[\left(\phi_{u}^{\dagger} \phi_{1}\right)\left(\phi_{d}^{\dagger} \phi_{1}\right)-\left(\phi_{u}^{\dagger} \phi_{2}\right)\left(\phi_{d}^{\dagger} \phi_{2}\right)\right]
\end{aligned}
$$

$$
\begin{aligned}
& +\lambda_{15}\left[\left(\phi_{u}^{\dagger} \phi_{1}\right)\left(\phi_{1}^{\dagger} \phi_{d}\right)-\left(\phi_{u}^{\dagger} \phi_{2}\right)\left(\phi_{2}^{\dagger} \phi_{d}\right)\right] \\
& +\lambda_{16}\left(\phi_{1}^{\dagger} \phi_{1}-\phi_{2}^{\dagger} \phi_{2}\right)\left(\phi_{u}^{\dagger} \phi_{d}\right) \\
& \left.+\lambda_{17}\left(\phi_{u}^{\dagger} \phi_{d}\right)^{2}+\text { h.c. }\right\}, \\
V_{\text {quadratic }}= & \mu_{11}^{2}\left(\phi_{1}^{\dagger} \phi_{1}\right)+\mu_{22}^{2}\left(\phi_{2}^{\dagger} \phi_{2}\right)+\mu_{u u}^{2}\left(\phi_{u}^{\dagger} \phi_{u}\right) \\
& +\mu_{d d}^{2}\left(\phi_{d}^{\dagger} \phi_{d}\right)+\mu_{12}^{2}\left(\phi_{1}^{\dagger} \phi_{2}+\text { h.c. }\right) \\
& +\mu_{1 u}^{2}\left(\phi_{1}^{\dagger} \phi_{u}+\text { h.c. }\right)+\mu_{1 d}^{2}\left(\phi_{1}^{\dagger} \phi_{d}+\text { h.c. }\right) \\
& +\mu_{2 u}^{2}\left(\phi_{2}^{\dagger} \phi_{u}+\text { h.c. }\right)+\mu_{2 d}^{2}\left(\phi_{2}^{\dagger} \phi_{d}+\text { h.c. }\right) \\
& +\mu_{u d}^{2}\left(\phi_{u}^{\dagger} \phi_{d}+\text { h.c. }\right) .
\end{aligned}
$$

We wish to reiterate that we have softly broken the $D_{4}$ symmetry by making $V_{\text {quadratic }}$ to be completely general, but $V_{\text {quartic }}$ obeys the $D_{4}$ symmetry. For simplicity, we take all the parameters to be real. Even then, above potential includes a large number of free parameters and its analysis can easily become quite involved. However, all we need to show is that (i) the scalar potential does not impose any restrictions on the relative hierarchies of the VEVs, and (ii) the nonstandard scalars may be safely decoupled from the EW scale. Thus, it will suffice if we can show that there exists an intuitive and convenient limit of the D4HDM scalar potential, which can easily accommodate these features. Such a limit can be obtained from Eq. (35), by setting $\lambda_{1,5,6} \rightarrow \lambda$ together with $\lambda_{7,8,9} \rightarrow 2 \lambda$ and the rest of the quartic parameters to zero. The resulting potential reads:

$$
\begin{aligned}
V_{\mathrm{MS}}= & \mu_{11}^{2} \phi_{1}^{\dagger} \phi_{1}+\mu_{22}^{2} \phi_{2}^{\dagger} \phi_{2}+\mu_{u u}^{2} \phi_{u}^{\dagger} \phi_{u}+\mu_{d d}^{2} \phi_{d}^{\dagger} \phi_{d} \\
& +\left(\mu_{12}^{2} \phi_{1}^{\dagger} \phi_{2}+\mu_{1 u}^{2} \phi_{1}^{\dagger} \phi_{u}+\mu_{1 d}^{2} \phi_{1}^{\dagger} \phi_{d}\right. \\
& \left.+\mu_{2 u}^{2} \phi_{2}^{\dagger} \phi_{u}+\mu_{2 d}^{2} \phi_{2}^{\dagger} \phi_{d}+\mu_{u d}^{2} \phi_{u}^{\dagger} \phi_{d}+\text { h.c. }\right) \\
& +\lambda\left(\phi_{1}^{\dagger} \phi_{1}+\phi_{2}^{\dagger} \phi_{2}+\phi_{u}^{\dagger} \phi_{u}+\phi_{d}^{\dagger} \phi_{d}\right)^{2},
\end{aligned}
$$

which has an enhanced symmetry in the quartic part of the potential. It is quite well-known that the two and three Higgsdoublet versions of such maximally symmetric potentials keep the VEV structure general and the nonstandard masses arise solely from the soft-breaking parameters, thereby making them easy to decouple $[26,29,30]$. From the counting of parameters, it is quite foreseeable that such general attributes will still be preserved for the maximally symmetric 4HDM potential displayed in Eq. (37).

To analyze the potential, we decompose the scalar doublets as in Eq. (31), and the ensuing tadpole equations can be used to express the four diagonal bilinears in terms of the 
four VEVs as follows

$$
\begin{aligned}
& \mu_{11}^{2}=-\frac{1}{2 v_{1}}\left(4 \lambda v^{2} v_{1}+\mu_{12}^{2} v_{2}+\mu_{1 u}^{2} v_{u}+\mu_{1 d}^{2} v_{d}\right), \\
& \mu_{22}^{2}=-\frac{1}{2 v_{2}}\left(4 \lambda v^{2} v_{2}+\mu_{12}^{2} v_{1}+\mu_{2 u}^{2} v_{u}+\mu_{1 d}^{2} v_{d}\right), \\
& \mu_{u u}^{2}=-\frac{1}{2 v_{u}}\left(4 \lambda v^{2} v_{u}+\mu_{1 u}^{2} v_{1}+\mu_{2 u}^{2} v_{2}+\mu_{u d}^{2} v_{d}\right), \\
& \mu_{d d}^{2}=-\frac{1}{2 v_{d}}\left(4 \lambda v^{2} v_{d}+\mu_{1 d}^{2} v_{1}+\mu_{2 d}^{2} v_{u}+\mu_{u d}^{2} v_{u}\right),
\end{aligned}
$$

where $v^{2}=v_{1}^{2}+v_{2}^{2}+v_{u}^{2}+v_{d}^{2}=(174 \mathrm{GeV})^{2}$ is the EW VEV. It should be noted that these equations pose no restrictions on the relative magnitudes of the VEVs. After the spontaneous symmetry breaking, we can extract the scalar mass matrices. Under our assumption of real parameters, the mass matrices of the scalars and pseudoscalars become disentangled. We now define the mass matrices for the different sectors as follows

$V_{S}^{\text {mass }}=\left(h_{1}, h_{2}, h_{u}, h_{d}\right) \frac{\mathcal{M}_{S}^{2}}{2}\left(\begin{array}{l}h_{1} \\ h_{2} \\ h_{u} \\ h_{d}\end{array}\right)$, where we have not shown the lower triangles of the matrices explicitly, because they are symmetric.

We will now parametrize four VEVs following a straightforward generalization of the $\tan \beta$ parameter for the 2HDM. Since all the scalar VEVs contribute to the EW VEV, we define:

$$
\begin{aligned}
& v_{1}=v \cos \beta \cos \beta_{1} \cos \beta_{2}, \quad v_{2}=v \sin \beta \cos \beta_{1} \cos \beta_{2} \\
& v_{u}=v \sin \beta_{1} \cos \beta_{2}, \quad v_{d}=v \sin \beta_{2},
\end{aligned}
$$

which satisfies $v_{1}^{2}+v_{2}^{2}+v_{u}^{2}+v_{d}^{2}=v^{2}$ by design, and $\tan \beta=v_{2} / v_{1}$ still holds. We can rotate to the Higgs basis by shifting all of the VEVs into a single scalar, which will have SM-like couplings to the fermions and gauge bosons,

$h=\frac{1}{v}\left(v_{1} h_{1}+v_{2} h_{2}+v_{u} h_{u}+v_{d} h_{d}\right)$.

As we will see shortly, this state $h$ automatically emerges as a physical mass eigenstate, thus ensuring the compatibility of our model with the measurements of the Higgs signalstrengths. A straightforward way to rotate to the Higgs basis will be to use the following orthogonal matrix

$$
O_{\beta}=\left(\begin{array}{cccc}
\cos \beta \cos \beta_{1} \cos \beta_{2} & \sin \beta \cos \beta_{1} \cos \beta_{2} & \sin \beta_{1} \cos \beta_{2} & \sin \beta_{2} \\
-\sin \beta & \cos \beta & 0 & 0 \\
-\cos \beta \sin \beta_{1} & -\sin \beta \sin \beta_{1} & \cos \beta_{1} & 0 \\
-\cos \beta \cos \beta_{1} \sin \beta_{2} & -\sin \beta \cos \beta_{1} \sin \beta_{2} & -\sin \beta_{1} \sin \beta_{2} \cos \beta_{2}
\end{array}\right)
$$

$$
V_{P}^{\text {mass }}=\left(z_{1}, z_{2}, z_{u}, z_{d}\right) \frac{\mathcal{M}_{P}^{2}}{2}\left(\begin{array}{c}
z_{1} \\
z_{2} \\
z_{u} \\
z_{d}
\end{array}\right),
$$

$V_{C}^{\text {mass }}=\left(\varphi_{1}^{+}, \varphi_{2}^{+}, \varphi_{u}^{+}, \varphi_{d}^{+}\right) \mathcal{M}_{C}^{2}\left(\begin{array}{l}\varphi_{1}^{-} \\ \varphi_{2}^{-} \\ \varphi_{u}^{-} \\ \varphi_{d}^{-}\end{array}\right)$.

Due to the particularly simple structure of the maximally symmetric potential, we find to obtain the mass matrices in the Higgs basis, as follows:

$$
\begin{aligned}
O_{\beta} \cdot \mathcal{M}_{S}^{2} \cdot O_{\beta}^{T} & =\left(\begin{array}{cc}
4 \lambda v^{2} & 0 \\
0 & \left(\mathcal{M}^{2}\right)_{3 \times 3}
\end{array}\right), \\
O_{\beta} \cdot \mathcal{M}_{P}^{2} \cdot O_{\beta}^{T} & =\left(\begin{array}{cc}
0 & 0 \\
0 & \left(\mathcal{M}^{2}\right)_{3 \times 3}
\end{array}\right), \\
O_{\beta} \cdot \mathcal{M}_{C}^{2} \cdot O_{\beta}^{T} & =\left(\begin{array}{cc}
0 & 0 \\
0 & \left(\mathcal{M}^{2}\right)_{3 \times 3}
\end{array}\right) .
\end{aligned}
$$

Indeed, the Higgs basis automatically block-diagonalizes the mass matrices, especially $\mathcal{M}_{S}^{2}$. Thus, from Eq. (45a), we

$$
\begin{aligned}
& \mathcal{M}_{P}^{2}=\left(\begin{array}{cccc}
-\frac{\mu_{12}^{2} v_{2}+\mu_{1 u}^{2} v_{u}+\mu_{1 d}^{2} v_{d}}{2 v_{1}} & \frac{\mu_{12}^{2}}{2} & \frac{\mu_{1 u}^{2}}{2} & \frac{\mu_{1 d}^{2}}{2} \\
\cdot & -\frac{\mu_{12}^{2} v_{1}+\mu_{2 u}^{2} v_{u}+\mu_{2 d}^{2} v_{d}}{2 v_{2}} & \frac{\mu_{2 u}^{2}}{2} & \frac{\mu_{2 d}^{2}}{2} \\
\cdot & \cdot & -\frac{\mu_{1 u}^{2} v_{1}+\mu_{2 u}^{2} v_{2}+\mu_{u d}^{2} v_{d}}{2 v_{u}} & \frac{\mu_{u d}^{2}}{2} \\
\cdot & \cdot & \cdot & -\frac{\mu_{1 d}^{2} v_{1}+\mu_{2 d}^{2} v_{2}+\mu_{u d}^{2} v_{u}}{2 v_{d}}
\end{array}\right) \\
& \mathcal{M}_{S}^{2}=\mathcal{M}_{P}^{2}+\left(\begin{array}{cccc}
4 \lambda v_{1}^{2} & 4 \lambda v_{1} v_{2} & 4 \lambda v_{1} v_{u} & 4 \lambda v_{1} v_{d} \\
\cdot & 4 \lambda v_{2}^{2} & 4 \lambda v_{2} v_{u} & 4 \lambda v_{2} v_{d} \\
\cdot & \cdot & 4 \lambda v_{u}^{2} & 4 \lambda v_{u} v_{d} \\
\cdot & \cdot & \cdot & 4 \lambda v_{d}^{2}
\end{array}\right), \quad \mathcal{M}_{C}^{2}=\mathcal{M}_{P}^{2},
\end{aligned}
$$


see that the scalar which features SM-like couplings to the fermions and gauge bosons is also a mass eigenstate of the theory, with squared-mass $m_{h}^{2}=4 \lambda v^{2}$. In other words, in this case we are automatically in the alignment limit, and the model comfortably accommodates an SM-like Higgs. Most importantly, the remaining $3 \times 3$ block matrices related to the nonstandard scalars are identical in the three sectors, and are independent of the EW scale, as they do not feature any dependence on $v$ :

$$
\begin{aligned}
&\left(\mathcal{M}^{2}\right)_{11}=-\frac{1}{2} \tan \beta\left[\operatorname { s i n } \beta \left(\mu_{12}^{2} \sin \beta\right.\right. \\
&\left.+\mu_{1 d}^{2} \sec \beta_{1} \tan \beta_{2}+\mu_{1 u}^{2} \tan \beta_{1}\right) \\
&+\cos \beta \cot ^{2} \beta\left(\mu_{2 d}^{2} \sec \beta_{1} \tan \beta_{2}+\mu_{2 u}^{2} \tan \beta_{1}\right) \\
&\left.+\mu_{12}^{2} \cos ^{2} \beta\left(\cot ^{2} \beta+2\right)\right] \\
&\left(\mathcal{M}^{2}\right)_{12}= \frac{1}{2}\left[\cos \beta\left(\mu_{2 d}^{2} \tan \beta_{1} \tan \beta_{2}+\mu_{2 u}^{2} \sec \beta_{1}\right)\right. \\
&\left.-\sin \beta\left(\mu_{1 d}^{2} \tan \beta_{1} \tan \beta_{2}+\mu_{1 u}^{2} \sec \beta_{1}\right)\right] \\
&\left(\mathcal{M}^{2}\right)_{13}= \frac{1}{2} \sec \beta_{2}\left(\mu_{2 d}^{2} \cos \beta-\mu_{1 d}^{2} \sin \beta\right) \\
&\left(\mathcal{M}^{2}\right)_{22}=-\frac{1}{2} \csc \beta_{1}\left\{\cos \beta_{1} \tan \beta_{2}\right. \\
& \times\left[\tan \beta_{1}\left(\mu_{1 d}^{2} \cos \beta+\mu_{2 d}^{2} \sin \beta\right)+\mu_{u d}^{2}\right] \\
&\left.+\sec \beta_{1}\left(\mu_{1 u}^{2} \cos \beta+\mu_{u d}^{2} \sin \beta\right)\right\} \\
&\left(\mathcal{M}^{2}\right)_{23}= \frac{1}{2} \sec \beta_{2}\left[\mu_{u d}^{2} \cos \beta_{1}\right. \\
&\left.-\sin \beta_{1}\left(\mu_{1 d}^{2} \cos \beta+\mu_{2 d}^{2} \sin \beta\right)\right] \\
&=-\frac{1}{2} \csc \beta_{2} \sec \beta_{2} \\
& \times\left(\mu_{1 d}^{2} \cos \beta \cos \beta_{1}+\mu_{2 d}^{2} \sin \beta \cos \beta_{1}\right. \\
&\left.+\mu_{u d}^{2} \sin \beta_{1}\right) \\
&\left(\mathcal{M}^{2}\right)_{33}
\end{aligned}
$$

Since these $3 \times 3$ matrices are identical in all sectors, it is clear that the maximally symmetric case will feature a tier-wise degeneracy, with 3 nonstandard mass scales, implying $M_{H_{i}}=M_{A_{i}}=M_{H_{i}^{ \pm}}=M_{i}$, where $H_{i}, A_{i}, H_{i}^{ \pm}$denote the $i$-th nonstandard scalar, pseudoscalar, and charged mass eigenstates, respectively. As these are disentangled from the EW scale, this limit features not only the automatic alignment limit, but also the decoupling limit, and the nonstandard masses can be taken to be as heavy as desired, without any bounds arising from unitarity. This is a consequence of having a single quartic parameter, $\lambda$, which will correspond to the SM quartic coupling.

To provide more detail, we note that $\mathcal{M}^{2}$ is a real, symmetric $3 \times 3$ matrix. Thus, it can be diagonalized by an orthogonal matrix with 3 rotation angles:

$$
\begin{aligned}
O_{\alpha}=\left(\begin{array}{ccc}
1 & 0 & 0 \\
0 & \cos \alpha_{3} & \sin \alpha_{3} \\
0 & -\sin \alpha_{3} & \cos \alpha_{3}
\end{array}\right) & \cdot\left(\begin{array}{ccc}
\cos \alpha_{2} & 0 & \sin \alpha_{2} \\
0 & 1 & 0 \\
-\sin \alpha_{2} & 0 & \cos \alpha_{2}
\end{array}\right) \\
& \left(\begin{array}{cccc}
\cos \alpha_{1} & \sin \alpha_{1} & 0 \\
-\sin \alpha_{1} & \cos \alpha_{1} & 0 \\
0 & 0 & 1
\end{array}\right)
\end{aligned}
$$

such that

$$
O_{\alpha} \cdot\left(\mathcal{M}^{2}\right)_{3 \times 3} \cdot O_{\alpha}^{T}=\operatorname{diag}\left(M_{1}^{2}, M_{2}^{2}, M_{3}^{2}\right) .
$$

The above equation will lead to 6 relations between the 6 physical parameters (the 3 nonstandard mass scales, $M_{1}, M_{2}, M_{3}$, and the 3 mixing angles, $\alpha_{1}, \alpha_{2}, \alpha_{3}$ present in $O_{\alpha}$ ), and the elements of $\mathcal{M}^{2}$. Note that, the elements of $\mathcal{M}^{2}$ are still independent because of the presence of the 6 off-diagonal soft-breaking parameters. Thus it is possible to adjust these parameters such that we find any desired nonstandard mixings and masses.

To sum up, we find that for the maximally symmetric case, in the presence of soft-breaking terms:

- The alignment limit emerges automatically (there is a mass eigenstate which is SM-like).

- The nonstandard masses are disentangled from the EW scale (the nonstandard masses are tier-wise degenerate and decoupled).

- There is no restriction on the relative hierarchies of the VEVs.

- Unitarity and boundedness from below constraints are trivially satisfied, because there is only one quartic parameter $\lambda$, which is related to the SM-like Higgs mass as $m_{h}^{2}=4 \lambda v^{2}$.

- The $\rho$-parameter is trivially satisfied by the tier-wise degeneracy of the masses [31,32].

Therefore, it should be evident that the maximally symmetric case, in the presence of soft-breaking terms, is phenomenologically viable. Now, since the more general $D_{4}$ potential of Eq. (35) encompasses the maximally symmetric limit of Eq. (37), then there is a finite range of parameter space where these same conclusions apply to the softly-broken $D_{4}$ case.

\section{References}

1. L. Wolfenstein, Parametrization of the Kobayashi-Maskawa Matrix. Phys. Rev. Lett. 51, 1945 (1983). https://doi.org/10.1103/ PhysRevLett.51.1945

2. N. Cabibbo, Unitary symmetry and leptonic decays. Phys. Rev. Lett. 10, 531 (1963). https://doi.org/10.1103/PhysRevLett.10.531

3. S.L. Glashow, J. Iliopoulos, L. Maiani, Weak interactions with Lepton-Hadron symmetry. Phys. Rev. D 2, 1285 (1970). https:// doi.org/10.1103/PhysRevD.2.1285 
4. M. Kobayashi, T. Maskawa, CP violation in the renormalizable theory of weak interaction. Prog. Theor. Phys. 49, 652 (1973). https://doi.org/10.1143/PTP.49.652

5. F.J. Botella, G.C. Branco, M.N. Rebelo, J.I. Silva-Marcos, What if the masses of the first two quark families are not generated by the standard model Higgs boson? Phys. Rev. D 94, 115031 (2016). https://doi.org/10.1103/PhysRevD.94.115031. arXiv:1602.08011

6. F. Feruglio, Pieces of the flavour puzzle. Eur. Phys. J. C 75, 373 (2015). https://doi.org/10.1140/epjc/s10052-015-3576-5. arXiv:1503.04071

7. Z.-Z. Xing, Quark mass hierarchy and flavor mixing puzzles. Int. J. Mod. Phys. A 29, 1430067 (2014). https://doi.org/10.1142/ S0217751X14300671. arXiv:1411.2713

8. Z.-Z. Xing, Flavor structures of charged fermions and massive neutrinos. Phys. Rept. 854, 1 (2020). https://doi.org/10.1016/j. physrep.2020.02.001. arXiv:1909.09610

9. A. Adulpravitchai, A. Blum, C. Hagedorn, A Supersymmetric D4 Model for mu-tau Symmetry. JHEP 03, 046 (2009). https://doi.org/ 10.1088/1126-6708/2009/03/046. arXiv:0812.3799

10. H. Ishimori, T. Kobayashi, H. Ohki, Y. Omura, R. Takahashi, M. Tanimoto, D(4) Flavor Symmetry for Neutrino Masses and Mixing. Phys. Lett. B 662, 178 (2008). https://doi.org/10.1016/j.physletb. 2008.03.007. arXiv:0802.2310

11. C. Hagedorn, R. Ziegler, $\mu-\tau$ Symmetry and Charged Lepton Mass Hierarchy in a Supersymmetric $D_{4}$ Model. Phys. Rev. D 82, 053011 (2010). https://doi.org/10.1103/PhysRevD.82.053011. arXiv: 1007.1888

12. D. Meloni, S. Morisi, E. Peinado, Stability of dark matter from the D4xZ2 flavor group. Phys. Lett. B 703, 281 (2011). https://doi.org/ 10.1016/j.physletb.2011.07.084. arXiv: 1104.0178

13. V.V. Vien, Neutrino mass and mixing in the 3-3-1 model with neutral leptons based on D4 flavor symmetry. Mod. Phys. Lett. A 29, 1450122 (2014). https://doi.org/10.1142/S0217732314501223

14. V.V. Vien, H.N. Long, The $D_{4}$ flavor symmery in 3-3-1 model with neutral leptons. Int. J. Mod. Phys. A 28, 1350159 (2013). https:// doi.org/10.1142/S0217751X13501595. arXiv:1312.5034

15. V.V. Vien, H.N. Long, Quark masses and mixings in an extension of the standard model with $\mathrm{D}_{4}$ flavor symmetry. Phys. Atom. Nucl. 81, 750 (2018). https://doi.org/10.1134/S1063778818060303

16. A.E. Cárcamo Hernández, C.O. Dib, U.J. Saldaña Salazar, When $\tan \beta$ meets all the mixing angles, Phys. Lett. B 809, 135750 (2020). https://doi.org/10.1016/j.physletb.2020.135750. arXiv:2001.07140

17. C. Bonilla, L.M.G. de la Vega, R. Ferro-Hernandez, N. Nath, E. Peinado, Neutrino phenomenology in a left-right $D_{4}$ symmetric model. Phys. Rev. D 102, 036006 (2020). https://doi.org/10.1103/ PhysRevD.102.036006. arXiv:2003.06444

18. H. Ishimori, T. Kobayashi, H. Ohki, Y. Shimizu, H. Okada, M. Tanimoto, Non-Abelian Discrete Symmetries in Particle Physics. Prog. Theor. Phys. Suppl. 183, 1 (2010). https://doi.org/10.1143/ PTPS.183.1. arXiv: 1003.3552
19. D. Das, Relating the Cabibbo angle to $\tan \beta$ in a two Higgs-doublet model. Phys. Rev. D 100, 075004 (2019). https://doi.org/10.1103/ PhysRevD.100.075004. arXiv:1908.03961

20. S. Antusch, J. Kersten, M. Lindner, M. Ratz, Running neutrino masses, mixings and CP phases: Analytical results and phenomenological consequences. Nucl. Phys. B 674, 401 (2003). https://doi. org/10.1016/j.nuclphysb.2003.09.050. arXiv:hep-ph/0305273

21. shape Particle Data Group collaboration, Review of Particle Physics. PTEP , 083 C01 (2020). https://doi.org/10.1093/ptep/ ptaa104

22. M.S. Chanowitz, M.A. Furman, I. Hinchliffe, Weak interactions of ultraheavy fermions. Nucl. Phys. B 153, 402 (1979). https://doi. org/10.1016/0550-3213(79)90606-0

23. D. Das, I. Saha, Alignment limit in three Higgs-doublet models. Phys. Rev. D 100, 035021 (2019). https://doi.org/10.1103/ PhysRevD.100.035021. arXiv: 1904.03970

24. M. Nebot and J.a.P. Silva, Self-cancellation of a scalar in neutral meson mixing and implications for the LHC. Phys. Rev. D 92, 085010 (2015). https://doi.org/10.1103/PhysRevD.92.085010. arXiv:1507.07941

25. G. Bhattacharyya, D. Das, Nondecoupling of charged scalars in Higgs decay to two photons and symmetries of the scalar potential. Phys. Rev. D 91, 015005 (2015). https://doi.org/10.1103/ PhysRevD.91.015005. arXiv:1408.6133

26. F. Faro, J.C. Romao, J.P. Silva, Nondecoupling in Multi-Higgs doublet models. Eur. Phys. J. C 80, 635 (2020). https://doi.org/10.1140/ epjc/s10052-020-8217-y. arXiv:2002.10518

27. S. Carrolo, J.C. Romão, J.P. Silva, F. Vazão, Symmetry and decoupling in multi-Higgs boson models. Phys. Rev. D 103, 075026 (2021). https://doi.org/10.1103/PhysRevD.103.075026. arXiv:2102.11303

28. C.D. Froggatt, H.B. Nielsen, Hierarchy of Quark Masses, Cabibbo Angles and CP Violation. Nucl. Phys. B 147, 277 (1979). https:// doi.org/10.1016/0550-3213(79)90316-X

29. N. Darvishi, A. Pilaftsis, Classifying Accidental Symmetries in Multi-Higgs Doublet Models. Phys. Rev. D 101, 095008 (2020). https://doi.org/10.1103/PhysRevD.101.095008. arXiv:1912.00887

30. M. Chakraborti, D. Das, M. Levy, S. Mukherjee, I. Saha, Prospects for light charged scalars in a three-Higgs-doublet model with Z3 symmetry. Phys. Rev. D 104, 075033 (2021). https://doi.org/10. 1103/PhysRevD.104.075033. arXiv:2104.08146

31. W. Grimus, L. Lavoura, O.M. Ogreid, P. Osland, A Precision constraint on multi-Higgs-doublet models. J. Phys. G 35, 075001 (2008). https://doi.org/10.1088/0954-3899/35/7/075001. arXiv:0711.4022

32. W. Grimus, L. Lavoura, O.M. Ogreid, P. Osland, The Oblique parameters in multi-Higgs-doublet models. Nucl. Phys. B 801, 81 (2008). https://doi.org/10.1016/j.nuclphysb.2008.04.019. arXiv: 0802.4353 